\title{
Reconstructing the metric of the local Universe from number counts observations
}

\author{
Sergio Andres Vallejo ${ }^{2,3}$, Antonio Enea Romano ${ }^{1,2,3}$ \\ ${ }^{1}$ Theoretical Physics Department, CERN, \\ CH-1211 Geneva 23, Switzerland \\ ${ }^{2}$ Instituto de Fisica, \\ Universidad de Antioquia, \\ A.A.1226, Medellin, Colombia \\ ${ }^{3}$ ICRANet, Piazza della Repubblica 10, \\ I-65122 Pescara
}

\begin{abstract}
Number counts observations available with new surveys such as the Euclid mission will be an important source of information about the metric of the Universe. We compute the low red-shift expansion for the energy density and the density contrast using an exact spherically symmetric solution in presence of a cosmological constant. At low red-shift the expansion is more precise than linear perturbation theory prediction. We then use the local expansion to reconstruct the metric from the monopole of the density contrast. We test the inversion method using numerical calculations and find a good agreement within the regime of validity of the red-shift expansion. The method could be applied to observational data to reconstruct the metric of the local Universe with a level of precision higher than the one achievable using perturbation theory.
\end{abstract}




\section{INTRODUCTION}

The standard cosmological model is based on the assumption that the Universe is homogeneous and isotropic on sufficiently larges scales, and is confirmed by different observations such as for example the cosmic microwave background (CMB) radiation [1] or of galaxy catalogues. However the presence of structure at smaller scales can affect local observations as it was shown in [2], and it is therefore important to understand its consequences. The effects of inhomogeneities on cosmological observables have been studied in different cases [2 25] such as dark energy, the luminosity distance [12, 13, 18] or the expansion scalar [26] . These effects are due to the fact that spatial inhomogeneities change the energy of photons, modifying the cosmological red-shift due to the Universe expansion. As a consequence some errors are produced in the estimation of parameters based on homogeneous cosmological models.

One important source of information about the Universe are galaxy catalogues since they allow to map the local density field. Since we can only measure the red-shift of astrophysical objects for which other distance measurement methods such as stellar parallax cannot be applied, it is important to take into account the effects of these inhomogeneities on the metric in order to compute self-consistently the density in red-shift space. This is particularly important when trying to determine the metric of the Universe. Different numerical [23, 27-32] and analytical [33] inversion methods have been developed in absence of the cosmological constant. More recently a numerical inversion for the luminosity distance in presence of the cosmological constant was derived in [34], but no method has been developed to reconstruct the metric from density observation in presence of a cosmological constant.

In this paper we develop for the first time a low red-shift analytical inversion method in presence of a cosmological constant to reconstruct the metric from the monopole of the density contrast, modeling the monopole of local structure with a spherically symmetric exact solution of Einstein fields equations.

The paper is organized as follows. In section II we show how we model the monopole of the local structure using an exact solution of Einstein field equations. In section III we compute the redshift expansion of the geodesics equations [26]. In section IV we calculate the density $\rho(z)$ and the density contrast $\delta(z)$. Finally in section $\mathrm{V}$ we develop an analytical method to determine the metric of the Universe from the density contrast. 


\section{MODELING THE LOCAL UNIVERSE}

In order to model the monopole component of the local structure we use the LTB solution [35-39]

$$
d s^{2}=-d t^{2}+\frac{R^{\prime}(t, r)^{2}}{1+2 E(r)} d r^{2}+R(t, r)^{2} d \Omega^{2},
$$

where $E(r)$ is an arbitrary function of $r, R$ is a function of the time coordinate $t$ and the radial coordinate $r$, and the partial derivative of this function with respect to $r$ is denoted as $R^{\prime}(t, r)=\partial_{r} R(t, r)$. It follows from the Einstein equations that

$$
\begin{aligned}
\left(\frac{\dot{R}}{R}\right)^{2} & =\frac{2 E(r)}{R^{2}}+\frac{2 M(r)}{R^{3}}+\frac{\Lambda}{3}, \\
\rho(t, r) & =\frac{2 M^{\prime}}{R^{2} R^{\prime}}
\end{aligned}
$$

where $M(r)$ is an arbitrary function of the radial cordinate $r$, we adopt a system of units in which $c=8 \pi G=1$, and we denote the partial derivative of $R$ with respect to $t$ as $\dot{R}=\partial_{t} R(t, r)$.

The analytical solution of eq.(2) can be derived [40, 41] if we introduce new functions $\rho_{0}(r)$ and $k(r)$, and a new coordinate $\eta=\eta(t, r)$ given by

$$
\begin{aligned}
\left.\frac{\partial \eta}{\partial t}\right|_{r} & =\frac{r}{R}=\frac{1}{a}, \\
\rho_{0}(r) & =\frac{6 M(r)}{r^{3}}, \\
k(r) & =-\frac{2 E(r)}{r^{2}} .
\end{aligned}
$$

Without any loss of generality we will adopt the coordinate system in which $\rho_{0}(r)$ is a constant, which is known as the FLRW gauge. Then we can express eq.(2) in the form

$$
\left(\frac{\partial a}{\partial \eta}\right)^{2}=-k(r) a^{2}+\frac{\rho_{0}}{3} a+\frac{\Lambda}{3} a^{4} .
$$

The solution to this equation can be written in the form [10]

$$
a(\eta, r)=\frac{\rho_{0}}{k(r)+3 \wp\left(\frac{\eta}{2} ; g_{2}(r), g_{3}(r)\right)} .
$$

where $\wp\left(x ; g_{2}, g_{3}\right)$ is the Weierstrass elliptic function and

$$
g_{2}(r)=\frac{4}{3} k(r)^{2}, \quad g_{3}(r)=\frac{4}{27}\left(2 k(r)^{3}-\Lambda \rho_{0}^{2}\right) .
$$


The relation between $t$ and $\eta$ can be found by integrating eq.(44) and is given by [26]

$$
t(\eta, r)=\frac{2 \rho_{0}}{3 \wp^{\prime}\left(\wp^{-1}\left(-\frac{k(r)}{3}\right)\right)}\left[\ln \left(\frac{\sigma\left(\frac{\eta}{2}-\wp^{-1}\left(-\frac{k(r)}{3}\right)\right)}{\sigma\left(\frac{\eta}{2}+\wp^{-1}\left(-\frac{k(r)}{3}\right)\right)}\right)+\eta \zeta\left(\wp^{-1}\left(-\frac{k(r)}{3}\right)\right)\right]
$$

where $\wp^{\prime}$ is the derivative of the Weierstrass' elliptic function $\wp$.

In terms of the new coordinate $\eta$ and the function $a(\eta, r)$ the radial null geodesic equations are given by [6]

$$
\begin{aligned}
& \frac{d \eta}{d z}=-\frac{\partial_{r} t(\eta, r)+G(\eta, r)}{(1+z) \partial_{\eta} G(\eta, r)} \\
& \frac{d r}{d z}=\frac{a(\eta, r)}{(1+z) \partial_{\eta} G(\eta, r)}
\end{aligned}
$$

where the function $G(\eta, r)$ has an explicit analytical form given by

$$
\begin{aligned}
G(\eta, r) & \equiv \frac{R^{\prime}(t(\eta, r), r)}{\sqrt{1-k(r) r^{2}}} \\
R^{\prime}(t(\eta, r), r) & =\partial_{r}(a(\eta, r) r)-a^{-1} \partial_{\eta}(a(\eta, r) r) \partial_{r} t(\eta, r) .
\end{aligned}
$$

Finally, in terms of the new coordinate system the density profile is given by

$$
\rho(\eta, r)=\rho(t(\eta, r), r)=\frac{\rho_{0}}{a(\eta, r)^{2} R^{\prime}(t(\eta, r), r)} .
$$

\section{ANALYTICAL APPROXIMATIONS}

In order to obtain a low-redshift formula for the density contrast and the density profile we expand the function $k(r)$ as

$$
k(r)=k_{0}+k_{1} r+k_{2} r^{2}+\ldots
$$

From the exact solution for $a(\eta, r)$ we can obtain an expansion for $t(\eta, r)$ according to

$$
t(\eta, r)=t_{0}(r)+a\left(\eta_{0}, r\right)\left(\eta-\eta_{0}\right)+\frac{1}{2} \partial_{\eta} a\left(\eta_{0}, r\right)\left(\eta-\eta_{o}\right)^{2}+\ldots
$$

where we defined $t_{0}(r)$ by

$$
t_{0}(r) \equiv t\left(\eta_{0}, r\right)
$$

The low red-shift Taylor expansion for the geodesic equations can be found in [13]. However in order to reconstruct correctly the metric all the quantities which depend on the coefficients 
of $k(r)$ appearing in the analytical approximations, such as $t_{0}^{\prime}(0)$ and $t_{0}^{\prime \prime}(0)$, must be written explicitly in terms of those coefficients. Therefore we introduce the dimensionless quantities $K_{n}$, given by $k_{n}=K_{n}\left(a_{0} H_{0}\right)^{n+2}$, and applying the chain rule for the derivatives of $t_{0}(r)$ it follows from eq.(10) and (18) that

$$
\begin{aligned}
t_{0}^{\prime}(0) & =\left.\frac{\partial t_{0}(r)}{\partial r}\right|_{r=0}=\left.\left(\frac{\partial t_{0}}{\partial k} \frac{\partial k}{\partial r}\right)\right|_{r=0}=a_{0} \alpha K_{1}, \\
t_{0}^{\prime \prime}(0) & =a_{0}\left(a_{0} H_{0}\right)\left(\beta K_{1}^{2}+2 \alpha K_{2}\right)
\end{aligned}
$$

where $\alpha$ and $\beta$ are dimensionless quantities given by

$$
\begin{aligned}
& \alpha=\left.\frac{\left(a_{0} H_{0}\right)^{3}}{a_{0}} \frac{\partial t_{0}}{\partial k}\right|_{k=k_{0}}, \\
& \beta=\left.\frac{\left(a_{0} H_{0}\right)^{5}}{a_{0}} \frac{\partial^{2} t_{0}}{\partial k^{2}}\right|_{k=k_{0}} .
\end{aligned}
$$

Now we can replace the expressions from eq.(19) and (20) into the low-redshift Taylor expansion for the geodesic equations given in [26]. We will consider the case in which $k_{0}=0$, which is enough to understand qualitatively the effects of the inhomogeneity, since this term corresponds to the homogeneous component of the curvature function $k(r)$. The results for the general case for not vanishing $k_{0}$ are given in Appendix B.

The expansion for $r(z)$ and $\eta(z)$ requires to first expand the geodesics equations and then to solve a complicated system of linear equations. We have used the Mathematica software for all the analytical calculations, writing some simplifying routines to express all the results in terms of $H_{0}$ and other dimensionless parameters given below. This ensures an immediate check of the dimensional correctness of the results and facilitate their physical interpretation. The simplifying routines allow to eliminate the elliptic functions and their derivatives according to the procedure explained in Appendix A. Without this automatic manipulations the formulae would be very cumbersome and difficult to derive. For the geodesics redshift expansion we get 
the coefficients

$$
\begin{aligned}
\eta_{1}= & -\frac{\alpha K_{1}+1}{a_{0} H_{0}}, \\
\eta_{2}= & \frac{1}{12 a_{0} H_{0} \Omega_{M}}\left[2 K_{1}^{2}\left(-4 \alpha+9 \alpha^{2}\left(\Omega_{M}-1\right) \Omega_{M}-3 \beta \Omega_{M}\right)+K_{1}\left(27 \alpha \Omega_{M}^{2}-12 \alpha \Omega_{M}-4\right)+\right. \\
& \left.+3 \Omega_{M}\left(3 \Omega_{M}-4 \alpha K_{2}\right)\right], \\
r_{1}= & \frac{1}{a_{0} H_{0}}, \\
r_{2}= & \frac{K_{1}\left(-18 \alpha \Omega_{M}^{2}+12 \alpha \Omega_{M}+4\right)-9 \Omega_{M}^{2}}{12 a_{0} H_{0} \Omega_{M}}, \\
r_{3}= & \frac{1}{72 a_{0} H_{0} \Omega_{\Lambda} \Omega_{M}^{2}}\left[2 K _ { 1 } ^ { 2 } \left(4 \zeta_{0}+\Omega_{\Lambda}\left(-27\left(8 \alpha^{2}+\beta\right) \Omega_{M}^{3}+18\left(3 \alpha^{2}-4 \alpha+\beta\right) \Omega_{M}^{2}+\right.\right.\right. \\
& \left.\left.+162 \alpha^{2} \Omega_{M}^{4}+36 \alpha \Omega_{M}+4\right)-6 \zeta_{0} \Omega_{M}+2 \Omega_{M}\right)+12 K_{1} \Omega_{\Lambda} \Omega_{M}^{2}\left(27 \alpha \Omega_{M}^{2}-24 \alpha \Omega_{M}-5\right)+ \\
& \left.+3 \Omega_{\Lambda} \Omega_{M}\left(K_{2}\left(-36 \alpha \Omega_{M}^{2}+24 \alpha \Omega_{M}+8\right)+3\left(9 \Omega_{M}-4\right) \Omega_{M}^{2}\right)\right] .
\end{aligned}
$$

It is important to observe that all these formulae have the correct dimensions, since all the relevant quantities have been expressed in dimensionless form, apart from the dimensionfull

prefactor $H_{0}^{-1}$. In the above equations we have introduced the parameters $a_{0}, H_{0}, \Omega_{M}, \Omega_{\Lambda}, T_{0}$ and $\zeta_{0}$ according to their corresponding definitions given in [10, 26]. As can be seen from these expressions the effects of the inhomogeneity start to show respectively at first order for $\eta(z)$ and second order for $r(z)$ as can be seen from the formluae we found.

\section{FORMULAE FOR THE DENSITY PROFILE AND DENSITY CONTRAST AT LOW REDSHIFT}

The density profile in redshift space is given by

$$
\rho(z)=\rho(\eta(z), r(z))
$$


and substituting the formulae for $\eta(z), r(z)$ we obtain after expanding to second order in redshift

$$
\begin{aligned}
\rho(z)= & 3 \Omega_{M} H_{0}^{2}+\rho_{1} z+\rho_{2} z^{2} \\
\rho_{1}= & H_{0}^{2}\left(4 K_{1}\left(3 \alpha \Omega_{M}+1\right)+9 \Omega_{M}\right) \\
\rho_{2}= & -\frac{H_{0}^{2}}{12 \Omega_{\Lambda} \Omega_{M}}\left[K _ { 1 } ^ { 2 } \left(\Omega_{\Lambda}\left(-18\left(25 \alpha^{2}-4 \alpha+5 \beta\right) \Omega_{M}^{2}+81 \alpha^{2} \Omega_{M}^{3}-300 \alpha \Omega_{M}-40\right)+\right.\right. \\
& \left.+20\left(\Omega_{M}-\zeta_{0}\right)\right)+18 K_{1} \Omega_{\Lambda} \Omega_{M}\left(3 \alpha \Omega_{M}^{2}+(2-24 \alpha) \Omega_{M}-8\right)+ \\
& \left.-12 \Omega_{\Lambda} \Omega_{M}\left(5 K_{2}\left(3 \alpha \Omega_{M}+1\right)+9 \Omega_{M}\right)\right]
\end{aligned}
$$

It is important to observe that all these formulae have the correct dimensions, since all the relevant quantities have been expressed in dimensionless form, apart from the dimensionfull prefactor $H_{0}^{2}$. Another important result is that $a_{0}$ does not appear anywhere, as expected, since the value of $a_{0}$ is arbitrary and physically observable quantities should not depend on it.

In order to derive an analytical approximation for the density contrast we must first define what is the background density. For any scalar $\Phi(t, r)$ we first define the sub-horizon volume average on constant time slices

$$
\begin{aligned}
\bar{\Phi}(t) & =\frac{\int \Phi(t, r) d V(t)}{\int d V(t)} \\
\int d V(t) & =\int_{0}^{r_{H o r}(t)} \frac{R(t, r)^{2} R^{\prime}(t, r)}{\sqrt{1-k(r) r^{2}}} d r
\end{aligned}
$$

where the upper limit of the integral $r_{H o r}(t)$ is the comoving horizon as a function of time, and determines the region of space causally connected with the central observer at time $t$. Note in fact that spatial averaging on super-horizon scales is not physically meaningful, since the effects of super-horizon structures are unobservable [5].

We can then evaluate $\bar{\Phi}(t)$ at the time $t(z)$ corresponding to a given redshift $z$, i.e. the time along null radial geodesics, and define the background value of $\Phi$ at redshift $z$ as

$$
\bar{\Phi}(z)=\bar{\Phi}(t(z))
$$

Applying this definition of background value to $\rho$ we can define the density contrast

$$
\delta(z)=\frac{\rho(z)}{\bar{\rho}(z)}-1 .
$$


If the size of the local inhomogeneity is sufficiently smaller than the volume over which the integral in eq.(32) is performed then $\bar{\rho}$ will get most of its contribution from the asymptotically homogeneous region and the average density will be well approximated by the asymptotic density

$$
\bar{\rho}(z)=\lim _{r \rightarrow \infty} \rho(t(z), r) .
$$

This is clearly true for compensated structures of any size, but it also applies to uncompensated structures whose size is sufficiently smaller than the asymptotic homogeneous region.

We can now re-write the background energy density as

$$
\begin{aligned}
\bar{\rho}(z) & =3\left(H_{0}^{b}\right)^{2} \Omega_{M}^{b}(1+z)^{3}, \\
H_{0}^{b} & =\bar{H}(0) \\
\Omega_{M}^{b} & =\frac{\bar{\rho}(0)}{3\left(H_{0}^{b}\right)^{2}} \\
\Omega_{\Lambda}^{b} & =1-\Omega_{M}^{b},
\end{aligned}
$$

where the upper-script ${ }^{b}$ stands for background and $H$ is the expansion scalar $H(t, r)$ [26]. Note that as a consistency check we have verified numerically that, as expected, for the central sub-horizon inhomogeneities we considered in this paper both $\bar{H}(z)$ and $\bar{\rho}(z)$ computed through the volume average defined in eq.(32) are in good agreement with eq.(36).

Expanding the density contrast we find

$$
\begin{aligned}
\delta(z)= & \delta_{0}+\delta_{1} z+\delta_{2} z^{2} \\
\delta_{0}= & \left(\frac{H_{0}}{H_{0}^{b}}\right)^{2} \frac{\Omega_{M}}{\Omega_{M}^{b}}-1 \\
\delta_{1}= & \left(\frac{H_{0}}{H_{0}^{b}}\right)^{2} \frac{4 K_{1}\left(3 \alpha \Omega_{M}+1\right)}{3 \Omega_{M}^{b}} \\
\delta_{2}= & -\left(\frac{H_{0}}{H_{0}^{b}}\right)^{2} \frac{1}{36 \Omega_{\Lambda} \Omega_{M} \Omega_{M}^{b}}\left[18 K_{1} \Omega_{\Lambda} \Omega_{M}^{2}\left(3 \alpha \Omega_{M}+2\right)-60 K_{2} \Omega_{\Lambda} \Omega_{M}\left(3 \alpha \Omega_{M}+1\right)+\right. \\
& \left.+K_{1}^{2}\left(\Omega_{\Lambda}\left(-18\left(25 \alpha^{2}-4 \alpha+5 \beta\right) \Omega_{M}^{2}+81 \alpha^{2} \Omega_{M}^{3}-300 \alpha \Omega_{M}-40\right)+20\left(\Omega_{M}-\zeta_{0}\right)\right)\right]
\end{aligned}
$$

It is easy to check that all these formulae have the correct dimensions because all the relevant quantities have been expressed in dimensionless form. 
As can be seen the first order coefficients of the expansion of $\rho(z)$ and $\delta(z)$ depend on $K_{1}$, while the second order depend on both $K_{1}$ and $K_{2}$.

The procedure to reduce the analytical formulae to this form is rather complicated since it involves to express wherever possible all the intermediate expressions in terms of physically meaningful quantities and we give more details about it in appendix A. The formulae for the case in which $K_{0}$ is different from zero are rather cumbersome and we give them in appendix B.

\section{A. Comparison with numerical and pertubative calculations}

In order to test the formulae we have derived in the previous section we consider inhomogeneities defined by a spatial curvature function $k(r)$ of this type

$$
k(r)= \pm \frac{r}{5}[1-\tanh (2 r)],
$$

which is plotted in fig.(11). We solve numerically eq.(21) and the radial null geodesic equations given in [42]. This type of function $k(r)$ is satisfying the assumption we made in the previous section that $k(0)=k_{0}=0$ and it corresponds to compensated structures making it easy to define background quantities according to eq.(36).

For the models we consider in this section we have $H_{0}^{b}=H_{0}$ and $\Omega_{M}^{b}=\Omega_{M}$. It also follows for these models that the curvature of the background solution is $k^{b}=\lim _{r \rightarrow \infty} k(r)=0$, which corresponds to a flat homogeneous Universe.
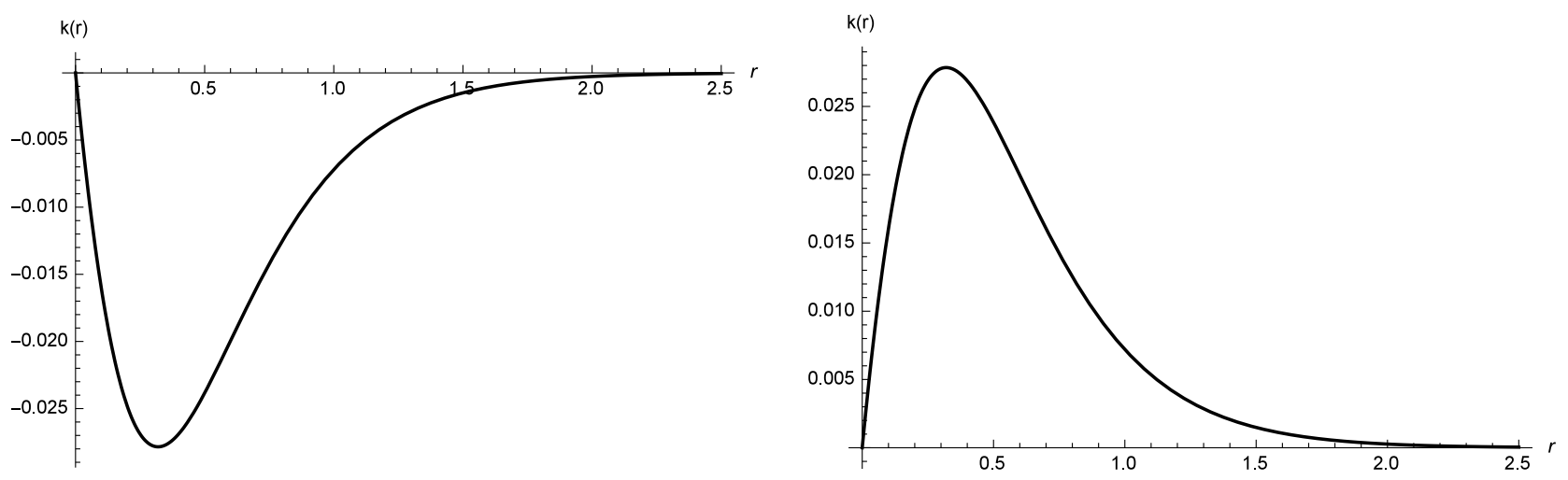

FIG. 1: The function $k(r)$ defined in eq.(45) is plotted in units of $H_{0}^{2}$ as a function of the radial coordinate in units of $H_{0}^{-1}$.

In order to compare our results to linear perturbation theory we compute the perturbation 
theory prediction for $\delta(z)$ according to [43]

$$
\delta(z) \approx-3 \delta H(z)\left(\Omega_{M}^{b}\right)^{-0.55}
$$

As can be seen in fig.(3) and fig.(2) at low red-shift the analytical formulae for $\rho(z)$ and $\delta(z)$ derived in eq.(29) and (41) are in good agreement with the numerical calculations and are more accurate than the perturbation theory prediction in eq.(46).
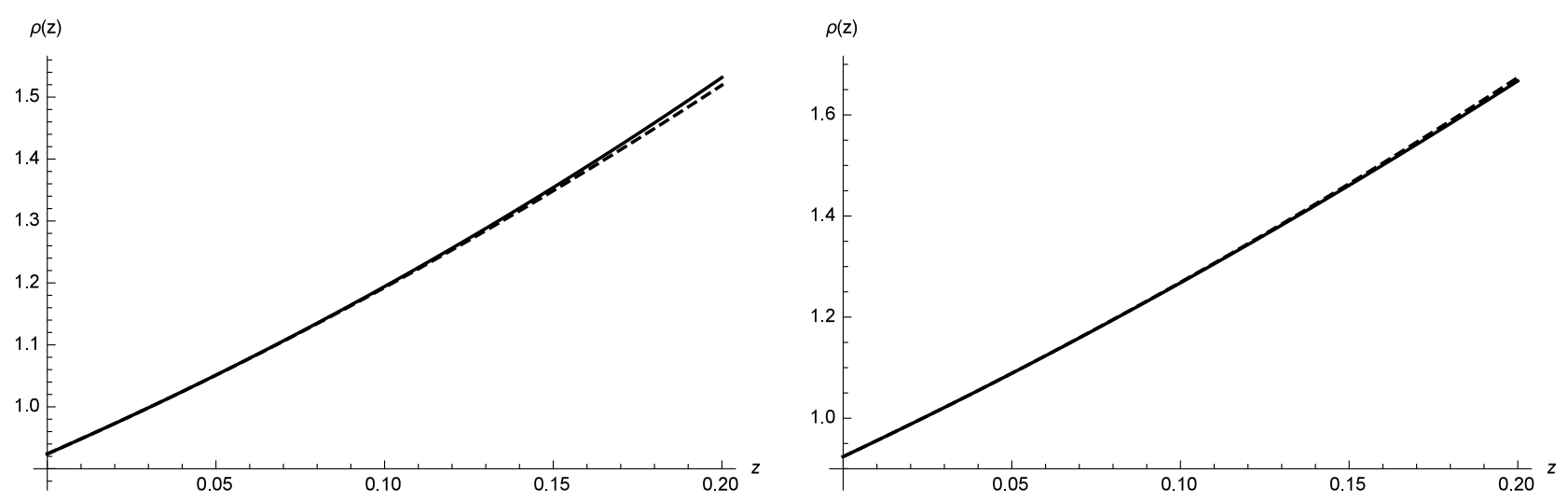

FIG. 2: The density profile in units of $H_{0}^{2}$ is plotted as a function of redshift. The left and right are plots are for the inhomogeneities corresponding to Fig. 1. The solid lines are for the numerical calculation and the dashed lines for the analytical approximation.
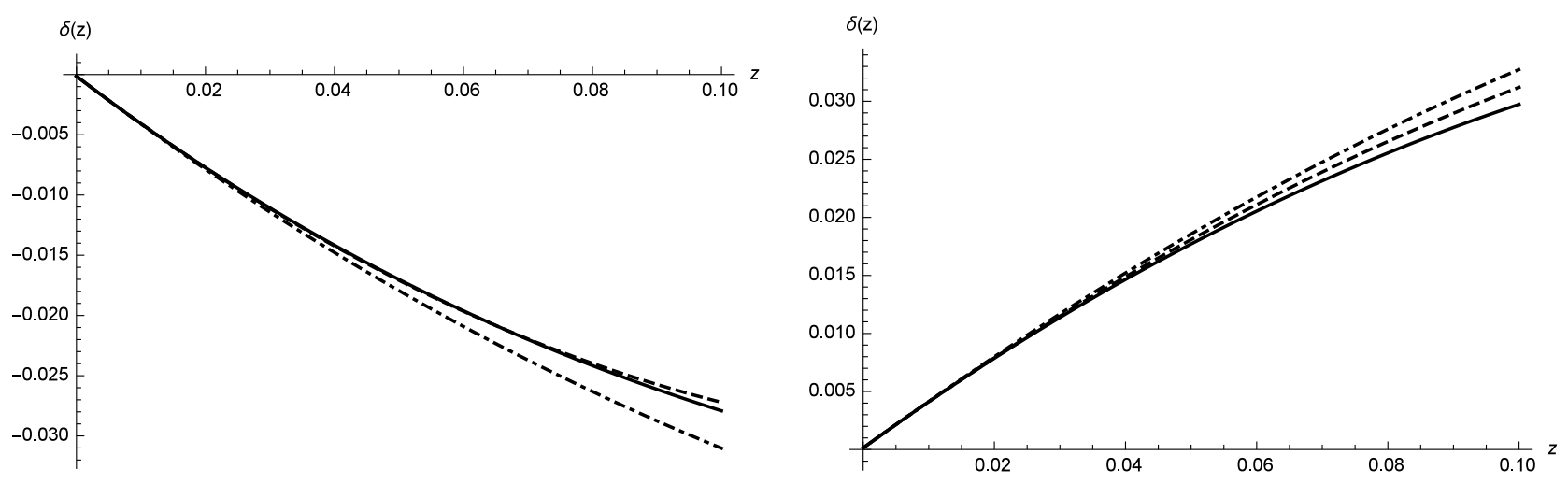

FIG. 3: The density contrast is plotted as a function of redshift. The left and right plots are for the inhomogeneities corresponding to Fig. 1. The solid lines correspond to the numerical solution, the dashed lines to the analytical formula we derived and the dot-dashed lines to the perturbation theory result. 

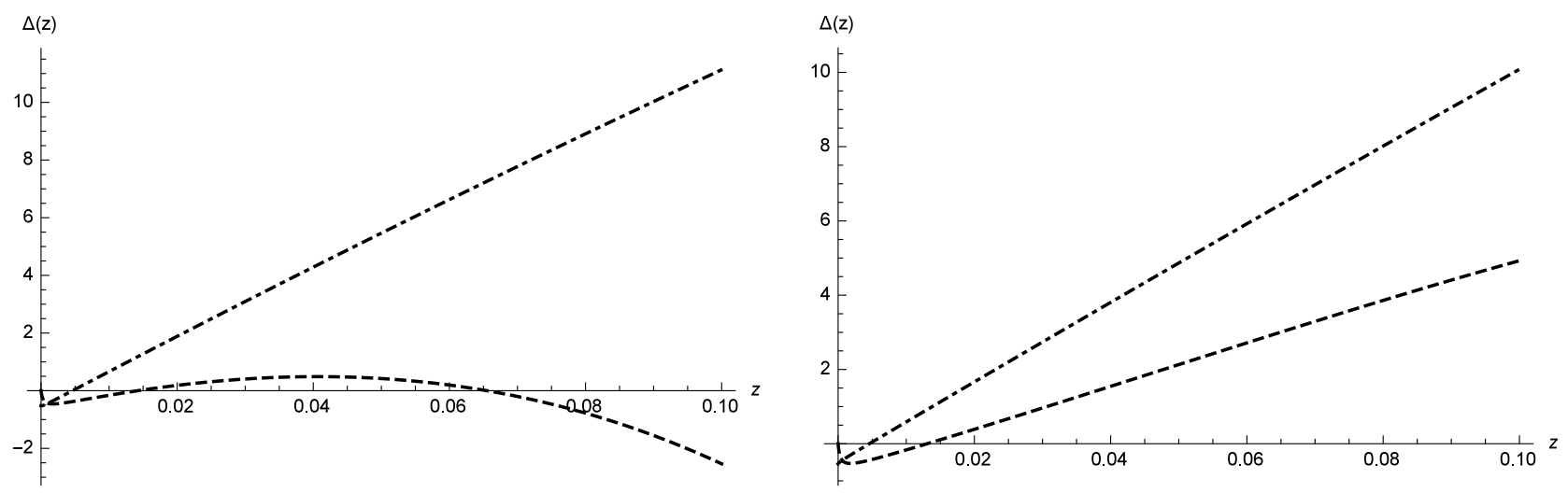

FIG. 4: The relative percentual difference between different analytical approximations and the numerical calculation $\Delta(z)=100\left(\frac{\delta^{A}(z)}{\delta^{N}(z)}-1\right)$ is plotted as a function of redshift. The left and right plots correspond to inhomogeneities in Fig. 1. The dashed lines are for the analytical formula and the dot-dashed lines for the perturbation theory approximation.

\section{RECONSTRUCTION OF THE METRIC FROM THE DENSITY CONTRAST}

In the previous section we have obtained the red-shift expansion of the density contrast in terms of the dimensionless coefficients $K_{i}$, and we will now use this to solve the inversion problem, i.e. to obtain $K_{i}$ from the density contrast. Note that in the coordinates we are using the coefficients $K_{i}$ complete determine the metric, so that we will be able to reconstruct the metric from the density contrast.

From eq.(43) and (44) we can solve the system of equations for the coefficient of the $k(r)$ expansion $K_{1}$ and $K_{2}$

$$
\begin{aligned}
k(r) \approx & K_{1}\left(a_{0} H_{0}\right)^{3} r+K_{2}\left(a_{0} H_{0}\right)^{4} r^{2} \\
K_{1}= & \left(\frac{H_{0}^{b}}{H_{0}}\right)^{2} \frac{3 \Omega_{M}^{b} \delta_{1}}{4\left(3 \alpha \Omega_{M}+1\right)} \\
K_{2}= & \left(\frac{H_{0}^{b}}{H_{0}}\right)^{4} \frac{3 \Omega_{M}^{b}}{320 \Omega_{\Lambda} \Omega_{M}\left(3 \alpha \Omega_{M}+1\right)^{3}}\left[8 \Omega _ { \Lambda } \Omega _ { M } ( 3 \alpha \Omega _ { M } + 1 ) \left(9 \alpha \delta_{1} \Omega_{M}^{2}+6\left(4 \alpha \delta_{2}+\delta_{1}\right) \Omega_{M}+\right.\right. \\
& \left.+8 \delta_{2}\right)+\delta_{1}^{2} \Omega_{M}^{b}\left(\frac{H_{0}}{H_{0}^{b}}\right)^{2}\left(\Omega_{\Lambda}\left(-18\left(25 \alpha^{2}-4 \alpha+5 \beta\right) \Omega_{M}^{2}+81 \alpha^{2} \Omega_{M}^{3}-300 \alpha \Omega_{M}-40\right)+\right. \\
& \left.\left.+20\left(\Omega_{M}-\zeta_{0}\right)\right)\right]
\end{aligned}
$$

It can be easily checked that all these formulae have the correct dimensions, since all the relevant quantities have been expressed in dimensionless form. 
The linear coefficient $K_{1}$ depends on $\delta_{1}$, while the second order coefficient $K_{2}$ depends on both $\delta_{1}$ and $\delta_{2}$. This is naturally expected since a homogeneous Universe corresponds to $K_{1}=K_{2}=\delta_{1}=\delta_{2}=0$. As a consistency check it can be easily verified that in fact $K_{1}=K_{2}=0$ in the homogeneous limit, i.e. when $\delta_{1}=\delta_{2}=0$.

It is important to note that the nonlinearity of Einsteins equations implies that the solution of the inversion problem (IP) is not unique. The input of the IP is in fact the monopole $\delta(z)$ of the density contrast, but the metric obtained applying to $\delta(z)$ the inversion is not necessarily the only possible solution of the IP. Other metrics with a different monopole and other higher multipoles could in fact produce the same $\delta(z)$. An additional degeneracy can come from inverting the metric using observations along the light cone.

The metric we obtain with our inversion method can be considered an effective one which does solve the inversion problem, but it is not the only possible solution, and becomes unique only in the linear limit when the effects of different multipoles can be decoupled or in the nonlinear regime under the a-priori assumption of spherical symmetry, i.e. in absence of any higher multipole. This degeneracy is related to the back-reaction effect due to the non commutativity of spatial averaging with the non linear differential operators present in the Einstein equations [44]. In the case of the Friedman equations back-reaction terms arise from spatial averaging, while in our case, when performing angular averages, the Einstein tensor of the monopole of the metric and the monopole of the Einstein tensor can differ by some analogous back-reaction term. The metric obtained by inversion from the monopole of the energy momentum tensor is an effective metric which includes some of these back-reactions terms, which are related to the angular average of higher multipoles. In fact the same degeneracy happens for the FLRW metric used as an effective description of the Universe on large scales, which can correspond to several different inhomogeneous metrics which all give the same effective FLRW metric after spatial averaging. In our case the effective metric has spherical symmetry, several anisotropic metrics could produce the same monopole of the Einstein tensor, corresponding to the same the same $\delta(z)$, and solve the IP.

The result of the inversion should thus be considered the monopole of the effective metric corresponding to assuming isotropy when angular averaging is performed on sufficiently large scales. In our case the homogeneity of the effective FLRW metric is replaced by the spherical symmetry of the LTB metric, which is supposed to be a well defined effective metric on sufficiently large angular scales, but on smaller scale this effective description may not be accurate. It should be noted in fact that if local structure were highly anisotropic this notion of effective 
metric may not be well defined, in the same way the FLRW effective description would not be very accurate if the Universe were highly inhomogeneous on all scales.

\section{A. Testing the accuracy of the inversion method}

In order to test the inversion method we compute numerically the density using the models defined in eq.(45) and then calculate the corresponding low red-shift expansion of $\delta(z)$. The coefficients of the reconstructed $k(r)$ are then obtained from eq.(47). The result of the inversion is then compared to the original $k(r)$ defined in eq.(45). As shown in fig.(5) at low red-shift the reconstructed $k(r)$ is in good agreement with the numerical results.

As shown in the previous section the perturbative calculation for $\delta(z)$ is less accurate than the analytical formula we computed in eq.(43,44). We can infer that also the pertubative solution of the inversion problem, which would consist in solving the perturbed Einstein equations to get the metric from the density contrast, will be less accurate than the analytical method we have developed.
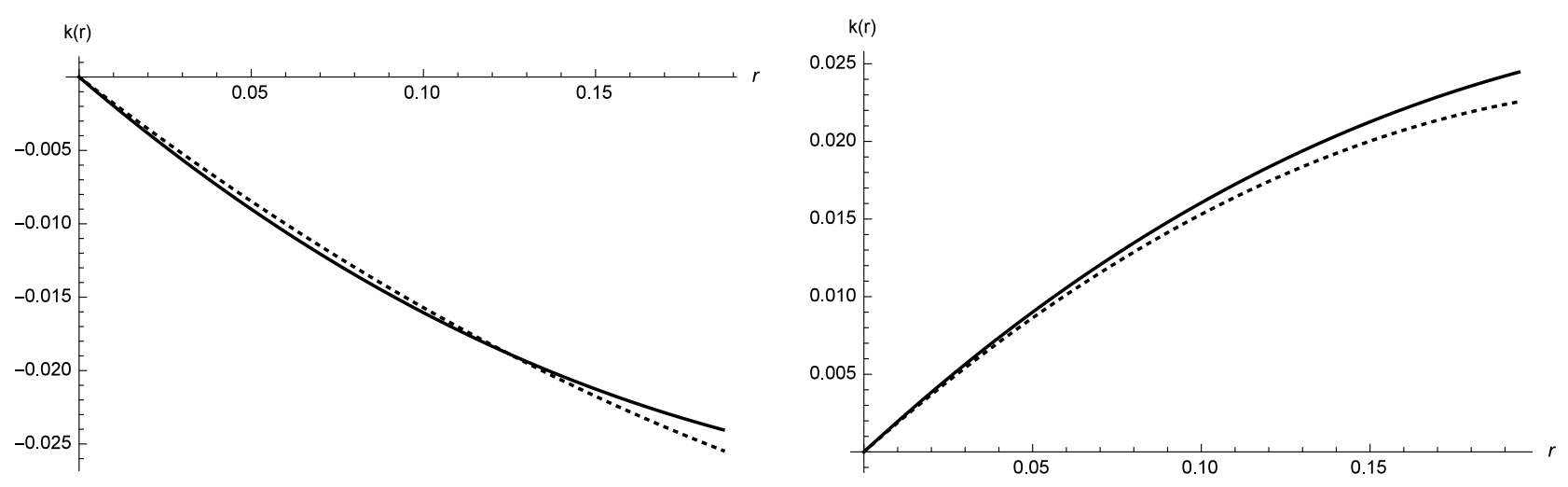

FIG. 5: The reconstructed metric function $k(r)$ is plotted in units of $H_{0}^{2}$ as a function of the radial coordinate in units of $H_{0}^{-1}$ for the inhomogeneities corresponding to Fig 1 . The black solid line corresponds to the original $k(r)$ function and the black dotted line to the reconstructed one.

\section{CONCLUSIONS}

We have derived the low-redshift expansion for the monopole of the density profile and the density contrast. At low red-shift the formulae are in good agreement with numerical solutions and are more accurate than the linear perturbation theory approximation. Using 
these formulae we have then developed a new analytical inversion method to reconstruct the metric from the monopole of the density contrast. The inversion method could be applied to low red-shift observational data to determine the metric with a level of precision higher than the one achievable using perturbation theory.

In the future the formulae we obtained for the metric could be used in the expansion of other cosmological observables to get coordinate independent formulas for these quantities in terms of the density contrast, without the need to expand the metric. It will also be interesting to develop a numerical inversion method able to reconstruct the metric beyond the regime of validity of the low red-shift expansion or to adopt other more accurate expansion techniques such as the Padé approximation. It will also be interesting to compare the results of the inversion method with perturbation theory methods in the Newton gauge used in modern galaxy analysis such as those given in [45]. This can be achieved by re-writing the LTB metric in the Newton gauge [46], and it would be important to check if the perturbative treatment including the effect of peculiar velocity and light propagation is in good agreement with our results or if other nonlinear effects can be important.

For a full reconstruction of the metric beyond the monopole contribution other solutions of the Einstein equations could be used for the analytical approach, in order to accommodate more complex geometries. For a general numerical inversion able to reconstruct any type of metric more sophisticated methods in numerical relativity will be required.

\section{Acknowledgments}

We thank the anonymous Referee for the suggestions to improve the manuscript and to follow up this paper with future projects about the comparison with other more accurate perturbative approaches.

\section{Appendix A: Derivation of the analytical formula}

In order to obtain the formulae for the red-shift expansion of $\rho(z)$ and $\delta(z)$ we have applied several manipulations and substitutions. The method is based on re-expressing everything in terms of physical quantities, starting from the definitions of $a_{0}$ and $H_{0}$, which are related to $\wp$ 
and $\wp^{\prime}$ by the equations

$$
\begin{aligned}
& a_{0} \equiv\left(\eta_{0}, 0\right)=\frac{\rho_{0}}{k_{0}+3 \wp_{0}}, \\
& H_{0} \equiv H\left(\eta_{0}, 0\right)=-\frac{3 \wp_{0}^{\prime}}{2 \rho_{0}},
\end{aligned}
$$

where

$$
\begin{aligned}
& \wp_{0}=\wp\left(\eta_{0} ; g_{2}(0), g_{3}(0)\right), \\
& \wp_{0}^{\prime}=\left.\frac{\partial \wp\left(\eta ; g_{2}(0), g_{3}(0)\right)}{\partial \eta}\right|_{\eta=\eta_{0}} .
\end{aligned}
$$

By inverting the previous equations we obtain the following relations

$$
\begin{aligned}
& \wp_{0}=\wp\left(\eta_{0} ; g_{2}(0), g_{3}(0)\right)=\frac{\rho_{0}-a_{0} k_{0}}{3 a_{0}}, \\
& \wp_{0}^{\prime}=\left.\frac{\partial \wp\left(\eta ; g_{2}(0), g_{3}(0)\right)}{\partial \eta}\right|_{\eta=\eta_{0}}=-\frac{2 H_{0} \rho_{0}}{3} .
\end{aligned}
$$

We can then substitute the above expressions everywhere $\wp$ and $\wp^{\prime}$ appear, making the final formula only depending on physical quantities such as $H_{0}$.

In order to simplify the results we have also used the Einstein equation for the LTB metric at the center $\left(\eta_{0}, 0\right)$

$$
1=-K_{0}+\Omega_{M}+\Omega_{\Lambda}
$$

and assumed a flat $\Lambda C D M$ for the background

$$
1=\Omega_{M}^{b}+\Omega_{\Lambda}^{b}
$$

\section{Appendix B: General formulae}

Here we give the low red-shift formulae for the density and for the solution to the inversion problem for the general case in which $k_{0}$ is different from zero. All the formulae are found using the computer algebra system provided by the Wolfram Mathematica software. We also test the accuracy of the general formulae against numerical calculations and linear perturbation theory for the density contrast. In order to do this comparisons we consider the type $\mathrm{I}^{-}$inhomogeneity studied in [13] 


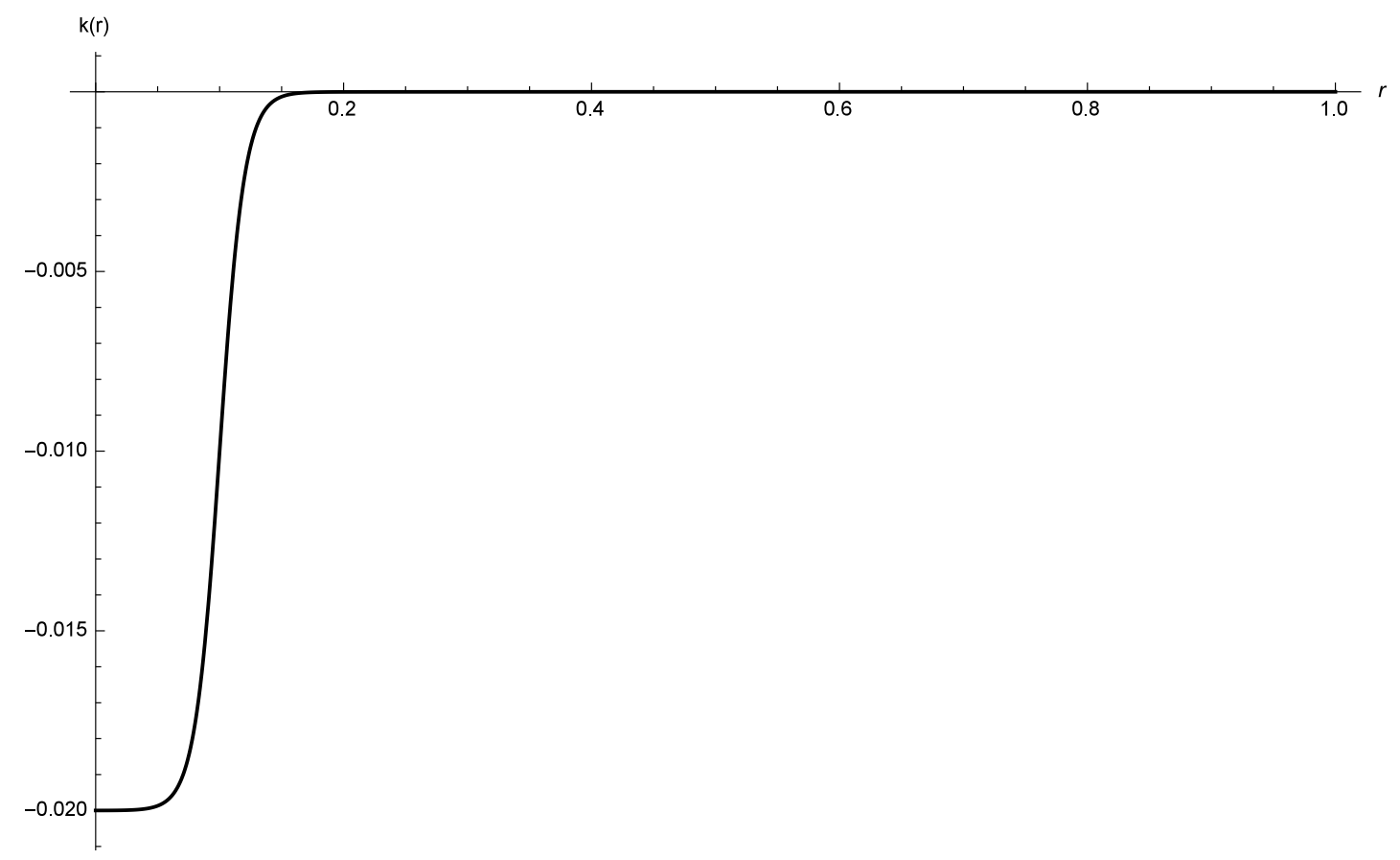

FIG. 6: The function $k(r)$ corresponding to the type $\mathrm{I}^{-}$inhomogeneity studied in [13] is plotted in units of $H_{0}^{2}$ as a function of the radial coordinate in units of $H_{0}^{-1}$. 
For the density profile we have

$$
\begin{aligned}
\rho(z)= & 3 H_{0}^{2} \Omega_{M}+\rho_{1} z+\rho_{2} z^{2}, \\
\rho_{1}= & \frac{3 H_{0}^{2} \Omega_{M}}{27 \Omega_{\Lambda} \Omega_{M}^{2}-4 K_{0}^{3}}\left[-4 K_{0}^{3}\left(4 \alpha K_{1}+3\right)+9 \Omega_{\Lambda} \Omega_{M}\left(4 K_{1}\left(3 \alpha \Omega_{M}+1\right)+9 \Omega_{M}\right)+\right. \\
& \left.+24 K_{1} K_{0}\left(\zeta_{0}-\Omega_{M}\right)+4 K_{1} K_{0}^{2}\left(T_{0}+2\right)\right] \\
\rho_{2}= & \frac{3 H_{0}^{2} \Omega_{M}}{4\left(4 K_{0}^{3}-27 \Omega_{M}^{2} \Omega_{\Lambda}\right)^{2}}\left[-16\left(K_{1}\left(T_{0}+4\right)-12\right) K_{0}^{6}+2\left(T_{0}\left(3 T_{0}+16\right) K_{1}^{2}+\right.\right. \\
& \left.+12\left(\left(T_{0}+12\right) \Omega_{M}-4\left(2 T_{0}+\zeta_{0}+4\right)\right) K_{1}-40 K_{2}\left(T_{0}+2\right)\right) K_{0}^{5}+\left(\left(8\left(24 \zeta_{0}+35\right)+\right.\right. \\
& \left.+T_{0}\left(8\left(5 T_{0}+9 \zeta_{0}+25\right)-9\left(T_{0}+16\right) \Omega_{M}\right)\right) K_{1}^{2}+144\left(\Omega_{M}\left(\zeta_{0}-2 \Omega_{M}-2 \Omega_{\Lambda}+9\right)+\right. \\
& \left.\left.-8 \zeta_{0}\right) K_{1}+480 K_{2}\left(\Omega_{M}-\zeta_{0}\right)\right) K_{0}^{4}+12\left(\left(18 \zeta_{0}^{2}+\left(40\left(T_{0}+3\right)-9\left(T_{0}+8\right) \Omega_{M}\right) \zeta_{0}+\right.\right. \\
& \left.+\Omega_{M}\left(T_{0}\left(12 \Omega_{M}+12 \Omega_{\Lambda}-43\right)-126\right)\right) K_{1}^{2}+9 \Omega_{M}\left(\left(T_{0}+8\right) \Omega_{M}-16\right) \Omega_{\Lambda} K_{1}+ \\
& \left.-12 \Omega_{M}\left(5 K_{2}+18 \Omega_{M}\right) \Omega_{\Lambda}\right) K_{0}^{3}-18\left(2 \left(-40 \zeta_{0}^{2}+6 \Omega_{M}^{2}\left(-4 \zeta_{0}+T_{0} \Omega_{\Lambda}-6\right)+\right.\right. \\
& \left.+\Omega_{M}\left(\zeta_{0}\left(9 \zeta_{0}+86\right)-\left(20 T_{0}+24 \zeta_{0}+75\right) \Omega_{\Lambda}+5\right)\right) K_{1}^{2}+9 \Omega_{M}^{2}\left(\left(T_{0}+12\right) \Omega_{M}+\right. \\
& \left.\left.-4\left(2 T_{0}+\zeta_{0}+4\right)\right) \Omega_{\Lambda} K_{1}-30 K_{2}\left(T_{0}+2\right) \Omega_{M}^{2} \Omega_{\Lambda}\right) K_{0}^{2}+ \\
& +\left(\left(\left(6 K_{0}-9 \Omega_{M}+50\right) \alpha^{2}+10 \beta\right) K_{1}^{2}+20 \alpha K_{2}\right)\left(4 K_{0}^{3}-27 \Omega_{M}^{2} \Omega_{\Lambda}\right){ }^{2}+ \\
& +108 \Omega_{M} \Omega_{\Lambda}\left(\left(40 \zeta_{0}+\left(5 T_{0}-12 \zeta_{0}-28\right) \Omega_{M}\right) K_{1}^{2}+30 K_{2}\left(\zeta_{0}-\Omega_{M}\right) \Omega_{M}+\right. \\
& \left.+9 \Omega_{M}\left(8 \zeta_{0}+\Omega_{M}\left(-\zeta_{0}+2 \Omega_{M}+2 \Omega_{\Lambda}-9\right)\right) K_{1}\right) K_{0}+324 \Omega_{M}^{2} \Omega_{\Lambda}\left(9\left(4-\Omega_{M}\right) \Omega_{M} \Omega_{\Lambda} K_{1}+\right. \\
& \left.+5\left(\zeta_{0}-\Omega_{M}+2 \Omega_{\Lambda}\right) K_{1}^{2}+3 \Omega_{M}\left(5 K_{2}+9 \Omega_{M}\right) \Omega_{\Lambda}\right)+2 \alpha K_{1}\left(4 K_{0}^{3}-27 \Omega_{M}^{2} \Omega_{\Lambda}\right)\left(8 K_{0}^{4}+\right. \\
& -2\left(K_{1}\left(3 T_{0}+8\right)+6\left(\Omega_{M}-8\right)\right) K_{0}^{3}+K_{1}\left(-50\left(T_{0}+2\right)-36 \zeta_{0}+9\left(T_{0}+8\right) \Omega_{M}\right) K_{0}^{2}+ \\
& +6 K_{1} \zeta_{0}\left(9 \Omega_{M}-50\right) K_{0}-6 \Omega_{M}\left(9 \Omega_{M} \Omega_{\Lambda}+K_{1}\left(12 \Omega_{M}+12 \Omega_{\Lambda}-53\right)\right) K_{0}+ \\
& \left.\left.\left.+8) \Omega_{M}+2 K_{1}\left(6 \Omega_{M}-25\right)\right) \Omega_{\Lambda}\right)\right] \\
& +3) \\
& \\
&
\end{aligned}
$$




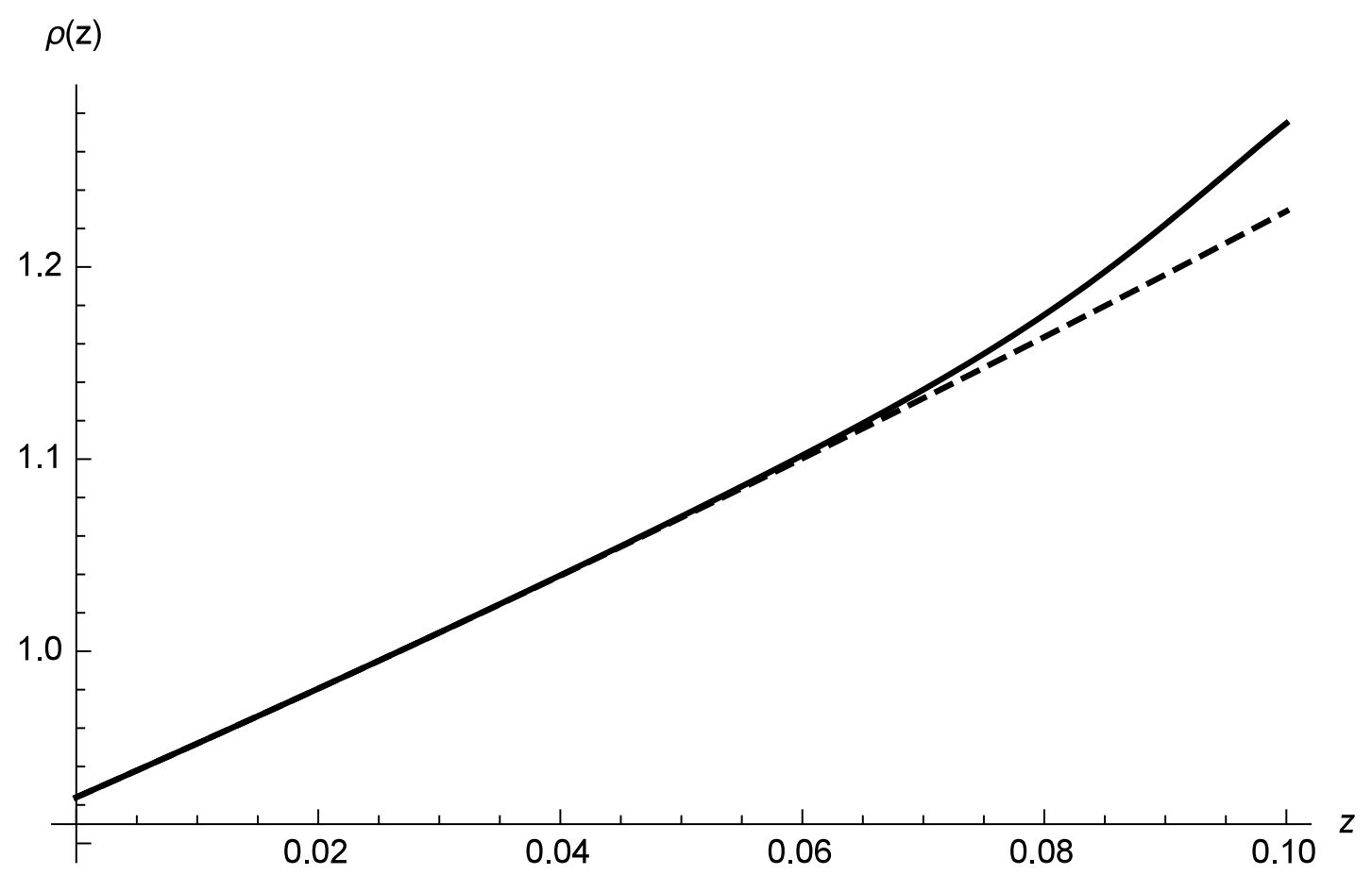

FIG. 7: The density profile in units of $H_{0}^{2}$ is plotted as a function of redshift for the type $\mathrm{I}^{-}$ inhomogeneity. The solid line is for the numerical calculation and the dashed line for the analytical approximation. 
The low red-shift expansion for the density contrast is

$$
\begin{aligned}
& \delta(z)=\delta_{0}+\delta_{1} z+\delta_{2} z^{2}, \\
& \delta_{0}=\left(\frac{H_{0}}{H_{0}^{b}}\right)^{2} \frac{\Omega_{M}}{\Omega_{M}^{b}}-1, \\
& \delta_{1}=-\left(\frac{H_{0}}{H_{0}^{b}}\right)^{2} \frac{4 K_{1} \Omega_{M}\left(-4 \alpha K_{0}^{3}-6 K_{0}\left(\Omega_{M}-\zeta_{0}\right)+K_{0}^{2}\left(T_{0}+2\right)+9 \Omega_{\Lambda} \Omega_{M}\left(3 \alpha \Omega_{M}+1\right)\right)}{\Omega_{M}^{b}\left(4 K_{0}^{3}-27 \Omega_{\Lambda} \Omega_{M}^{2}\right)}, \\
& \delta_{2}=\left(\frac{H_{0}}{H_{0}^{b}}\right)^{2} \frac{\Omega_{M}}{4 \Omega_{M}^{b}\left(4 K_{0}^{3}-27 \Omega_{\Lambda} \Omega_{M}^{2}\right)^{2}}\left[( 4 K _ { 0 } ^ { 3 } - 2 7 \Omega _ { \Lambda } \Omega _ { M } ^ { 2 } ) ^ { 2 } \left(20 \alpha K_{2}+K_{1}^{2}(10 \beta+\right.\right. \\
& \left.\left.+\alpha^{2}\left(6 K_{0}-9 \Omega_{M}+50\right)\right)\right)+324 \Omega_{\Lambda} \Omega_{M}^{2}\left(5 K_{1}^{2}\left(\zeta_{0}+2 \Omega_{\Lambda}-\Omega_{M}\right)-9 K_{1} \Omega_{\Lambda} \Omega_{M}^{2}+\right. \\
& \left.+15 K_{2} \Omega_{\Lambda} \Omega_{M}\right)+2 \alpha K_{1}\left(4 K_{0}^{3}-27 \Omega_{\Lambda} \Omega_{M}^{2}\right)\left(-6 K_{0}\left(K _ { 1 } \left(50 \zeta_{0}+\right.\right.\right. \\
& \left.\left.+\Omega_{M}\left(-9 \zeta_{0}+12 \Omega_{\Lambda}-53\right)+12 \Omega_{M}^{2}\right)+9 \Omega_{\Lambda} \Omega_{M}^{2}\right)+9 \Omega_{\Lambda} \Omega_{M}\left(2 K_{1}\left(6 \Omega_{M}-25\right)+9 \Omega_{M}^{2}\right)+ \\
& \left.+K_{1} K_{0}^{2}\left(9\left(T_{0}+8\right) \Omega_{M}-2\left(18 \zeta_{0}+25 T_{0}+50\right)\right)-2 K_{0}^{3}\left(K_{1}\left(3 T_{0}+8\right)+6 \Omega_{M}\right)+8 K_{0}^{4}\right)+ \\
& +K_{0}^{4}\left(-144 K_{1} \Omega_{M}\left(-\zeta_{0}+2 \Omega_{\Lambda}+2 \Omega_{M}-1\right)+480 K_{2}\left(\Omega_{M}-\zeta_{0}\right)+K_{1}^{2}\left(8\left(24 \zeta_{0}+35\right)+\right.\right. \\
& \left.\left.-8 T_{0}\left(-9 \zeta_{0}+18 \Omega_{M}-25\right)+T_{0}^{2}\left(40-9 \Omega_{M}\right)\right)\right)+12 K_{0}^{3}\left(-60 K_{2} \Omega_{\Lambda} \Omega_{M}+\right. \\
& +K_{1}^{2}\left(\Omega_{M}\left(T_{0}\left(-9 \zeta_{0}+12 \Omega_{\Lambda}-43\right)-18\left(4 \zeta_{0}+7\right)\right)+12 T_{0} \Omega_{M}^{2}+2 \zeta_{0}\left(9 \zeta_{0}+20 T_{0}+60\right)\right)+ \\
& \left.+9 K_{1}\left(T_{0}+8\right) \Omega_{\Lambda} \Omega_{M}^{2}\right)-18 K_{0}^{2}\left(2 K _ { 1 } ^ { 2 } \left(-40 \zeta_{0}^{2}+6 \Omega_{M}^{2}\left(-4 \zeta_{0}+T_{0} \Omega_{\Lambda}-6\right)+\right.\right. \\
& \left.+\Omega_{M}\left(9 \zeta_{0}^{2}+86 \zeta_{0}-\Omega_{\Lambda}\left(24 \zeta_{0}+20 T_{0}+75\right)+5\right)\right)+9 K_{1} \Omega_{\Lambda} \Omega_{M}^{2}\left(\left(T_{0}+12\right) \Omega_{M}-4 \zeta_{0}\right)+ \\
& \left.-30 K_{2}\left(T_{0}+2\right) \Omega_{\Lambda} \Omega_{M}^{2}\right)+108 K_{0} \Omega_{\Lambda} \Omega_{M}\left(9 K_{1} \Omega_{M}^{2}\left(-\zeta_{0}+2 \Omega_{\Lambda}+2 \Omega_{M}-1\right)+\right. \\
& \left.+30 K_{2} \Omega_{M}\left(\zeta_{0}-\Omega_{M}\right)+K_{1}^{2}\left(40 \zeta_{0}+\Omega_{M}\left(-12 \zeta_{0}+5 T_{0}-28\right)\right)\right)+ \\
& +K_{0}^{5}\left(2 K_{1}\left(-48 \zeta_{0}+K_{1} T_{0}\left(3 T_{0}+16\right)+12\left(T_{0}+12\right) \Omega_{M}\right)-80 K_{2}\left(T_{0}+2\right)\right)+ \\
& \left.-16 K_{1} K_{0}^{6}\left(T_{0}+4\right)\right] \text {. }
\end{aligned}
$$




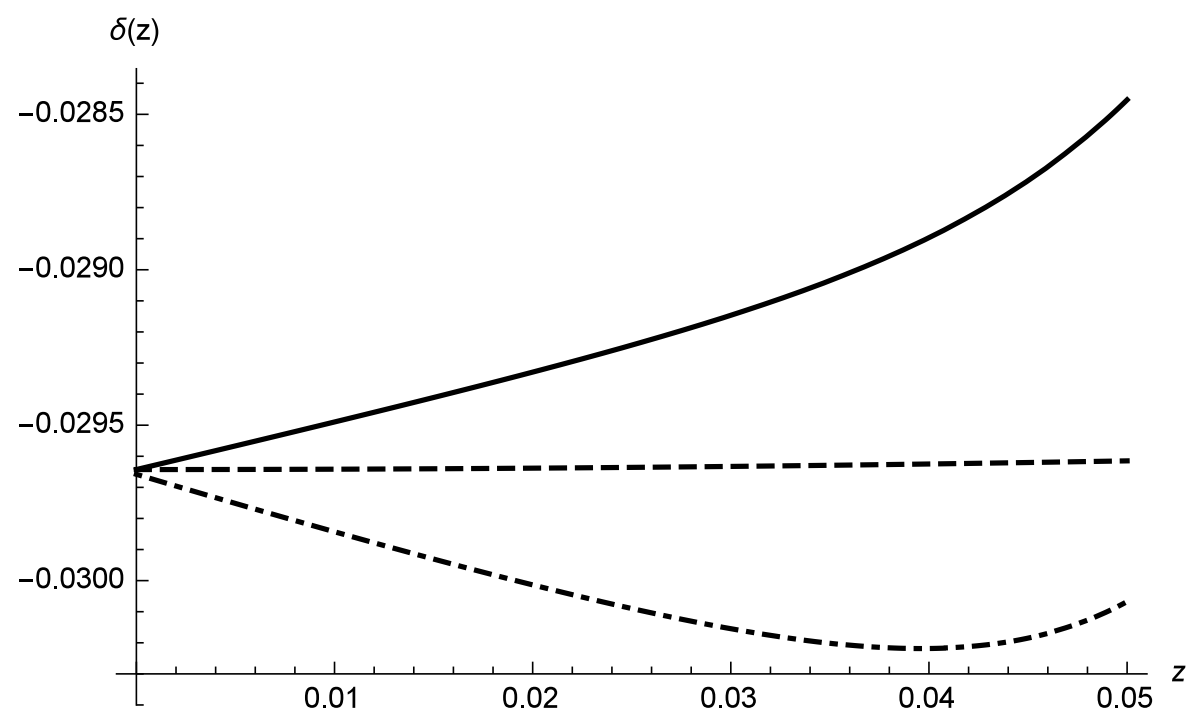

FIG. 8: The density contrast is plotted as a function of redshift for the type $\mathrm{I}^{-}$ inhomogeneity. The solid line corresponds to the numerical solution, the dashed line to the analytical formula we derived and the dot-dashed line to the perturbation theory result.

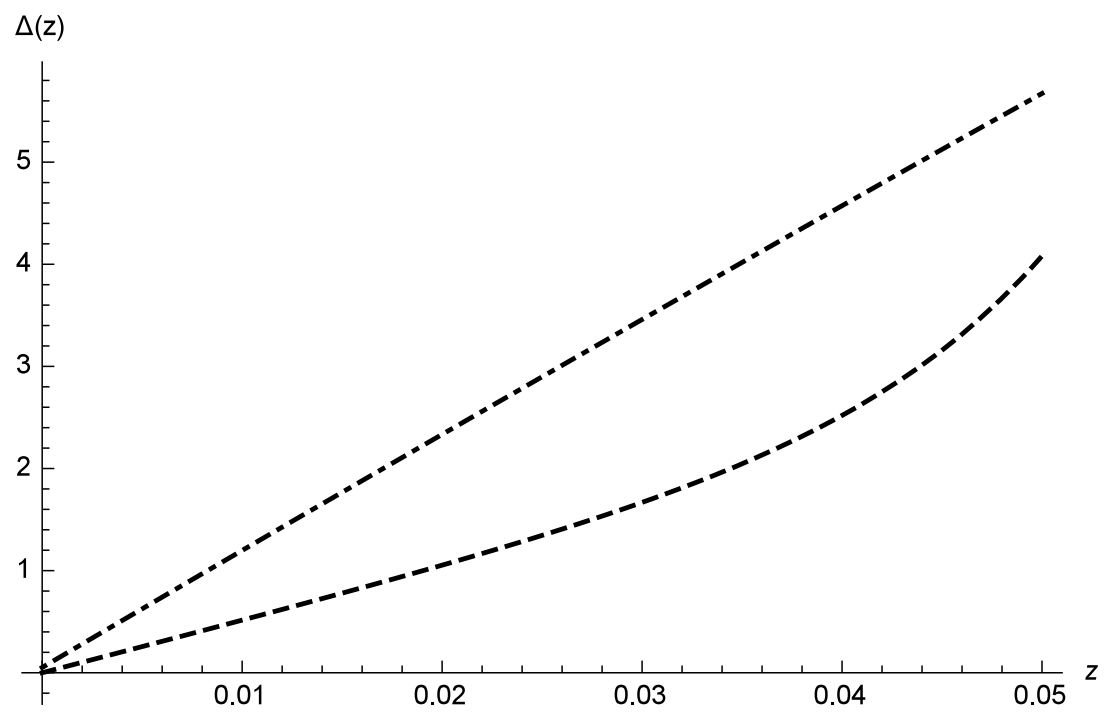

FIG. 9: The relative percentual difference between different analytical approximations and the numerical calculation $\Delta(z)=100\left(\frac{\delta^{A}(z)}{\delta^{N}(z)}-1\right)$ is plotted as a function of redshift for the type $\mathrm{I}^{-}$inhomogeneity. The dashed line is for the analytical formula and the dot-dashed line for the perturbation theory approximation. 
The solution of the inversion problem is

$$
\begin{aligned}
& k(r)=\left(a_{0} H_{0}\right)^{2} K_{0}+\left(a_{0} H_{0}\right)^{3} K_{1} r+\left(a_{0} H_{0}\right)^{4} K_{2} r^{2}, \\
& K_{1}=\left(\frac{H_{0}^{b}}{H_{0}}\right)^{2} \frac{\delta_{1} \Omega_{M}^{b}\left(27 \Omega_{\Lambda} \Omega_{M}^{2}-4 K_{0}^{3}\right)}{4 \Omega_{M}\left(-4 \alpha K_{0}^{3}-6 K_{0}\left(\Omega_{M}-\zeta_{0}\right)+K_{0}^{2}\left(T_{0}+2\right)+9 \Omega_{\Lambda} \Omega_{M}\left(3 \alpha \Omega_{M}+1\right)\right)}, \\
& K_{2}=\left(\frac{H_{0}^{b}}{H_{0}}\right)^{4} \frac{\Omega_{M}^{b}}{320 \Omega_{M}^{2}} \mathcal{A}\left[8 \Omega _ { M } \left(4 \alpha K_{0}^{3}-\left(T_{0}+2\right) K_{0}^{2}+6\left(\Omega_{M}-\zeta_{0}\right) K_{0}+\right.\right. \\
& \left.-9 \Omega_{M}\left(3 \alpha \Omega_{M}+1\right) \Omega_{\Lambda}\right)\left(8 \alpha \delta_{1} K_{0}^{4}-2\left(16 \alpha \delta_{2}+\delta_{1}\left(T_{0}+6 \alpha \Omega_{M}+4\right)\right) K_{0}^{3}+\right. \\
& +\left(4\left(2\left(T_{0}+2\right) \delta_{2}-3 \delta_{1} \zeta_{0}\right)+3\left(T_{0}+12\right) \delta_{1} \Omega_{M}\right) K_{0}^{2}-6\left(3 \delta_{1}\left(3 \alpha \Omega_{\Lambda}+2\right) \Omega_{M}^{2}+\right. \\
& \left.+\left(8 \delta_{2}+\delta_{1}\left(-3 \zeta_{0}+6 \Omega_{\Lambda}-3\right)\right) \Omega_{M}-8 \delta_{2} \zeta_{0}\right) K_{0}+9 \Omega_{M}\left(9 \alpha \delta_{1} \Omega_{M}^{2}+\right. \\
& \left.\left.+6\left(\delta_{1}+4 \alpha \delta_{2}\right) \Omega_{M}+8 \delta_{2}\right) \Omega_{\Lambda}\right)\left(\frac{H_{0}}{H_{0}^{b}}\right)^{2}+\delta_{1}^{2} \Omega_{M}^{b}\left(96 \alpha^{2} K_{0}^{7}-16\left(\left(9 \Omega_{M}-50\right) \alpha^{2}+\right.\right. \\
& \left.+\left(3 T_{0}+8\right) \alpha-10 \beta\right) K_{0}^{6}+\left(6 T_{0}^{2}+8\left(\alpha\left(9 \Omega_{M}-50\right)+4\right) T_{0}+\right. \\
& \left.+32 \alpha\left(-9 \zeta_{0}+18 \Omega_{M}-25\right)\right) K_{0}^{5}+\left(\left(40-9 \Omega_{M}\right) T_{0}^{2}-8\left(-9 \zeta_{0}+18 \Omega_{M}-25\right) T_{0}+\right. \\
& \left.+8\left(-18 \alpha\left(9 \alpha \Omega_{\Lambda}+4\right) \Omega_{M}^{2}+6 \alpha\left(53-12 \Omega_{\Lambda}\right) \Omega_{M}+6 \zeta_{0}\left(\alpha\left(9 \Omega_{M}-50\right)+4\right)+35\right)\right) K_{0}^{4}+ \\
& +12\left(162 \alpha^{2} \Omega_{\Lambda} \Omega_{M}^{3}+3\left(T_{0}\left(9 \alpha \Omega_{\Lambda}+4\right)-12\left(25 \alpha^{2}-4 \alpha+5 \beta\right) \Omega_{\Lambda}\right) \Omega_{M}^{2}+\right. \\
& \left.+\left(T_{0}\left(-9 \zeta_{0}+12 \Omega_{\Lambda}-43\right)-6\left(12 \zeta_{0}+50 \alpha \Omega_{\Lambda}+21\right)\right) \Omega_{M}+2 \zeta_{0}\left(20 T_{0}+9 \zeta_{0}+60\right)\right) K_{0}^{3}+ \\
& -18\left(27 \alpha\left(T_{0}+8\right) \Omega_{\Lambda} \Omega_{M}^{3}-6\left(8 \zeta_{0}+\left(25 T_{0} \alpha+18 \zeta_{0} \alpha+50 \alpha-2 T_{0}\right) \Omega_{\Lambda}+12\right) \Omega_{M}^{2}+\right. \\
& \left.+2\left(9 \zeta_{0}^{2}+86 \zeta_{0}-\left(20 T_{0}+24 \zeta_{0}+75\right) \Omega_{\Lambda}+5\right) \Omega_{M}-80 \zeta_{0}^{2}\right) K_{0}^{2}+ \\
& +54 \Omega_{M} \Omega_{\Lambda}\left(9 \alpha\left(9 \alpha \Omega_{\Lambda}+8\right) \Omega_{M}^{3}+6 \alpha\left(-9 \zeta_{0}+12 \Omega_{\Lambda}-53\right) \Omega_{M}^{2}+\right. \\
& \left.+2\left(5 T_{0}+150 \alpha \zeta_{0}-12 \zeta_{0}-28\right) \Omega_{M}+80 \zeta_{0}\right) K_{0}-81 \Omega_{M}^{2} \Omega_{\Lambda}\left(20\left(\Omega_{M}-\zeta_{0}\right)+\right. \\
& \left.\left.\left.+\left(81 \alpha^{2} \Omega_{M}^{3}-18\left(25 \alpha^{2}-4 \alpha+5 \beta\right) \Omega_{M}^{2}-300 \alpha \Omega_{M}-40\right) \Omega_{\Lambda}\right)\right)\right],
\end{aligned}
$$

where

$$
\mathcal{A}=\frac{\left(4 K_{0}^{3}-27 \Omega_{M}^{2} \Omega_{\Lambda}\right)}{\left(-4 \alpha K_{0}^{3}+\left(T_{0}+2\right) K_{0}^{2}-6\left(\Omega_{M}-\zeta_{0}\right) K_{0}+9 \Omega_{M}\left(3 \alpha \Omega_{M}+1\right) \Omega_{\Lambda}\right)^{3}}
$$




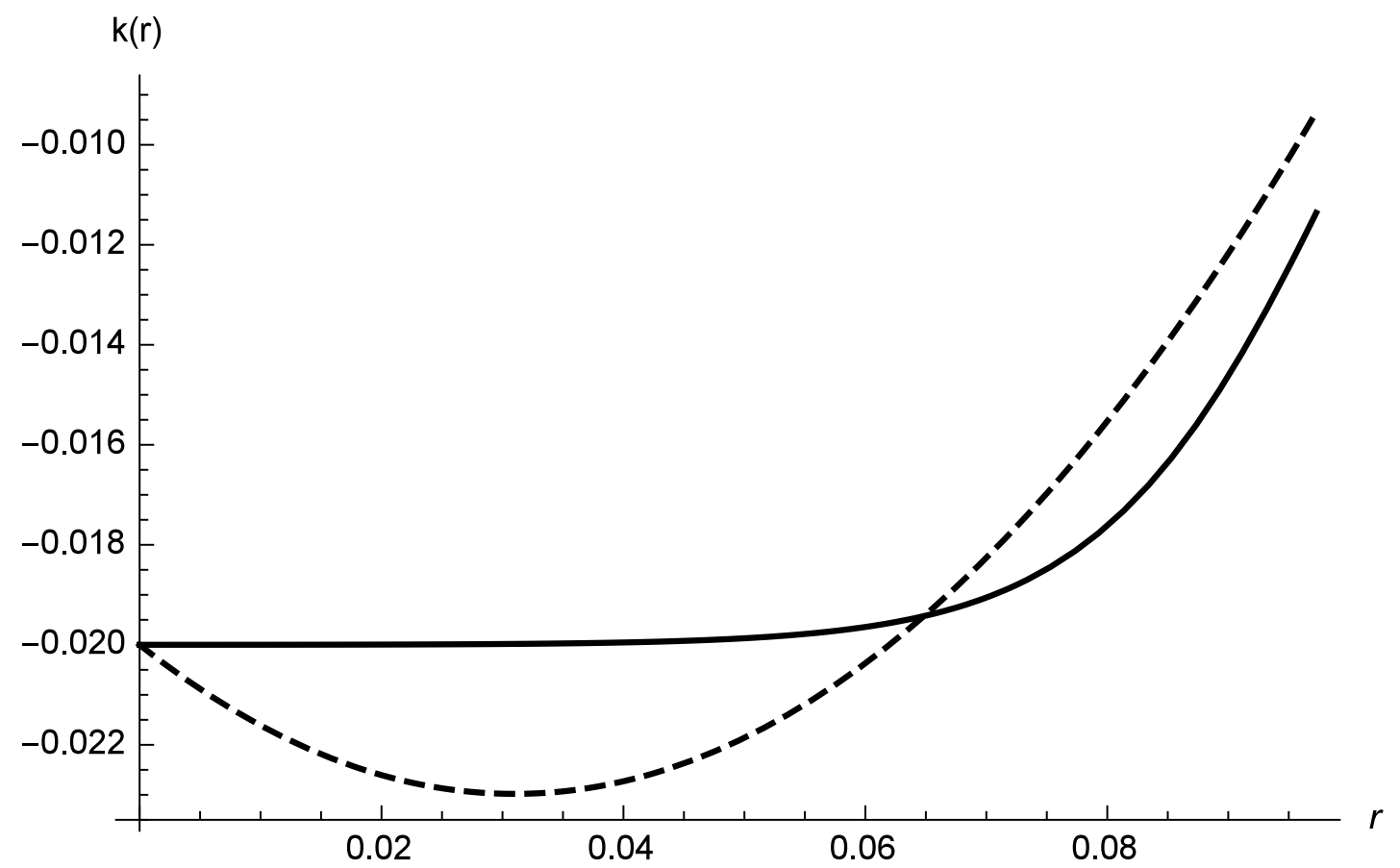

FIG. 10: The reconstructed metric function $k(r)$ is plotted in units of $H_{0}^{2}$ as a function of the radial coordinate in units of $H_{0}^{-1}$ for the type $\mathrm{I}^{-}$inhomogeneity. The black solid line corresponds to the original $k(r)$ function and the black dotted line to the reconstructed one.

\section{Acknowledgments}

[1] Planck Collaboration et al., Astronomy \& Astrophysics 594, A16 (2016), arXiv:1506.07135.

[2] A. Enea Romano and S. Andrés Vallejo, EPL (Europhysics Letters) 109, 39002 (2015), arXiv:1403.2034.

[3] A. E. Romano, S. Sanes, and M. Sasaki, (2013), arXiv:1311.1476.

[4] C. Clarkson and M. Regis, JCAP 1102, 013 (2011), arXiv:1007.3443.

[5] A. E. Romano, Phys. Rev. D75, 043509 (2007), arXiv:astro-ph/0612002.

[6] A. E. Romano and M. Sasaki, Gen.Rel.Grav. 44, 353 (2012), arXiv:0905.3342.

[7] I. Ben-Dayan, R. Durrer, G. Marozzi, and D. J. Schwarz, Phys.Rev.Lett. 112, 221301 (2014), arXiv:1401.7973.

[8] M. Redlich, K. Bolejko, S. Meyer, G. F. Lewis, and M. Bartelmann, Astron.Astrophys. 570, A63 (2014), arXiv:1408.1872. 
[9] A. E. Romano, JCAP 1001, 004 (2010), arXiv:0911.2927.

[10] A. E. Romano, Int.J.Mod.Phys. D21, 1250085 (2012), arXiv:1112.1777.

[11] V. Marra and A. Notari, Class.Quant.Grav. 28, 164004 (2011), arXiv:1102.1015.

[12] A. E. Romano and P. Chen, JCAP 1110, 016 (2011), arXiv:1104.0730.

[13] A. E. Romano, M. Sasaki, and A. A. Starobinsky, Eur.Phys.J. C72, 2242 (2012), arXiv:1006.4735.

[14] A. Krasiski, Phys.Rev. D90, 023524 (2014), arXiv:1405.6066.

[15] A. E. Romano, Gen.Rel.Grav. 45, 1515 (2013), arXiv:1206.6164.

[16] A. E. Romano, Int.J.Mod.Phys. D21, 1250085 (2012), arXiv:1112.1777.

[17] A. Balcerzak and M. P. Dabrowski, (2013), arXiv:1310.7231.

[18] A. E. Romano and P. Chen, Eur.Phys.J. C74, 2780 (2014), arXiv:1207.5572.

[19] A. E. Romano and M. Sasaki, General Relativity and Gravitation 44, 353 (2012), arXiv:0905.3342.

[20] G. Fanizza, M. Gasperini, G. Marozzi, and G. Veneziano, JCAP 1311, 019 (2013), arXiv:1308.4935.

[21] Romano, Antonio Enea and Chen, Pisin, Eur. Phys. J. C 74, 2780 (2014).

[22] A. Krasiski, Phys.Rev. D90, 103525 (2014), arXiv:1409.5377.

[23] D. J. H. Chung and A. E. Romano, Phys. Rev. D74, 103507 (2006), arXiv:astro-ph/0608403.

[24] A. E. Romano, H.-W. Chiang, and P. Chen, Class.Quant.Grav. 31, 115008 (2014).

[25] V. Marra, L. Amendola, I. Sawicki, and W. Valkenburg, Phys.Rev.Lett. 110, 241305 (2013), arXiv:1303.3121.

[26] A. E. Romano and S. A. Vallejo, European Physical Journal C 76, 216 (2016), arXiv:1502.07672.

[27] A. E. Romano, H.-W. Chiang, and P. Chen, Class. Quant. Grav. 31, 115008 (2014), arXiv:1312.4458.

[28] M.-N. Celerier, K. Bolejko, and A. Krasinski, Astron. Astrophys. 518, A21 (2010), arXiv:0906.0905.

[29] C.-M. Yoo, T. Kai, and K.-i. Nakao, Prog. Theor. Phys. 120, 937 (2008), arXiv:0807.0932.

[30] K. Bolejko, C. Hellaby, and A. H. A. Alfedeel, JCAP 1109, 011 (2011), arXiv:1102.3370.

[31] M. L. McClure and C. Hellaby, Phys. Rev. D78, 044005 (2008), arXiv:0709.0875.

[32] N. Mustapha, C. Hellaby, and G. F. R. Ellis, Mon. Not. Roy. Astron. Soc. 292, 817 (1997), arXiv:gr-qc/9808079.

[33] A. E. Romano, Phys. Rev. D82, 123528 (2010), arXiv:0912.4108. 
[34] M. Tokutake and C.-M. Yoo, JCAP 1610, 009 (2016), arXiv:1603.07837.

[35] G. Lemaître, Annales de la Société Scientifique de Bruxelles 53 (1933).

[36] G. Lemaitre, Gen.Rel.Grav. 29, 641 (1997).

[37] G. Lemaitre, Mon. Not. Roy. Astron. Soc. 91, 490 (1931).

[38] R. C. Tolman, Proc.Nat.Acad.Sci. 20, 169 (1934).

[39] H. Bondi, Mon. Not. Roy. Astron. Soc. 107, 410 (1947).

[40] A. Zecca, Adv.Stud.Theor.Phys. 7, 1101 (2013).

[41] D. Edwards, Monthly Notices of the Royal Astronomical Society 159, 51 (1972).

[42] M.-N. Celerier, Astron.Astrophys. 353, 63 (2000), arXiv:astro-ph/9907206.

[43] E. L. Turner, R. Cen, and J. P. Ostriker, Astrophys.J. 103, 1427 (1992).

[44] T. Buchert, Gen. Rel. Grav. 32, 105 (2000), arXiv:gr-qc/9906015.

[45] C. Bonvin and R. Durrer, Phys.Rev.D 84, 063505 (2011), arXiv:1105.5280.

[46] K. Van Acoleyen, Journal of Cosmology and Astroparticle Physics 10, 028 (2008), arXiv:0808.3554. 